\title{
The Design and Construction of an Intelligent Taxi Electronic Payment System Solution
}

\author{
Mozhgan Sadeghian ${ }^{1}$, Soheila Dadjou ${ }^{1}$, Majid Meghdadi ${ }^{1}$, Leila Safari ${ }^{1}$, Somayyeh Sadeghian ${ }^{2}$ \\ ${ }^{1}$ Computer Department, University of Zanjan, Zanjan, Zanjan, Iran \\ ${ }^{2}$ University of Birjand, Birjand, Iran \\ E-mail:mozhgan.sadeghian@gmail.com, sepandarmaz67@yahoo.com, \\ \{meghdad,__safari\}@znu.ac.ir, sadeghian2005@gmail.com \\ Received July 21, 2011; revised August 12, 2011; accepted September 10, 2011
}

\begin{abstract}
Today the usage of electronic services in different taxies seems to be necessary. Organizing a system to pay the taxi electronically with the card by the passenger can decrease the need to carry cash. Designing and building of such a system by using a special taximeter with new features and also one or more card reader can be fixed into the taxi. Payment is with the smart credit card and from the client's account. The information of presented services is stored in the system and will be recorded as offered services. The driver can have access to taxi organization or some special terminals electronically to see his account and also he can receive a receipt.
\end{abstract}

Keywords: Electronic Smart Card, Automation of Payment, Intelligent System

\section{Introduction}

Today according to the increasing population there are lots of problems in money payment to the taxi in cash. For example there is not enough access to cash and the other problem is damages to bill and as a result huge amount of budget to replace damaged and old bills.

In addition paying money and waiting for receiving the rest from the driver wastes time of the both passenger and the drive and of course enhances the traffic. These increase the fuel consumption and there is no effective control on the prices. So a new kind of technology is needed to manage all these disorders and problems. For this reason human was in the sake of a solution, at first they used electronic payment system for bus transporter system. Now it is time for taxi driving system. At this time more facilities are more likely to be served to passengers. In the second part of this paper we will have a review on the same projects. Then in the section three the methods and technologies used in the system will be discussed, in the section four the structure of hardware is presented. In the section five the facilities and privileges and at the end results and some suggestions are mentioned.

Since that paper [1] was published, there have been few technological changes, but progress has been made in consolidation and integration within the industry. This paper will briefly look at the current status of electronic payment systems, and progress in the last two years to widen the appeal and use of such systems [2].

\section{Related Works}

The statistics related to electronic business and electronic payment all around the world and the rate of its development is the importance of their role which they play in modern economy [3]. In Iran, some activities have been done in the field of electronics especially in electronic payment. A similar project which is present in Iran is electronic payment system in bus and train transporter system by using credit cards. Also in New York City an intelligent taxi payment in order to pay the portage electronically is used. The new GPS enabled New York City taxis includes a discussion of paying with credit cards or debit cards rather than cash. There are also some brief screen shots of the passenger interface. As with all electronic payment schemes, generational differences in payment preferences come into play: the older couple featured notes that "We'll pay the taxi with cash only because we always have" [4,5].

\section{Method and Technology Used in the System}

In this part of the article, the various kinds of smart cards are briefly introduced and then RFID technology and 
Mifare cards which are used in this project are mentioned.

\subsection{Objective of the System}

This project is about designing of an intelligent taxi payment system solution. Project's primary objective is to enhance the automation of payment in taxi and thus reach the some aims like not to wasting the time. In order to achieve this, we have set-up a small electronic payment system which facilitates the payment system. In the continuing section, system objects and their relations are described.

\subsection{Smart Cards}

A smart card, magnetic card or integrated circuit card, is a pocket card that ICs are put in it and are able to process data, it shows that the card can receive input data processed in the IC card and send it as output. Some kinds of intelligent cards are: contact smart card, contact less smart card, planar and coded.

Contact smart cards have contact region. When the card is put in the reader system the part contacts with electrical joiner which can read the information from the chip and then write.

In the smart cards the chip and card reader are related by the usage of RFID induction technology without any contact. These cards need almost one interference antenna to do their interactions. They are used usually when interactions should be processed quickly or with no hand meddle. So in order to design this model, Mifare S50 is used. Planar cards make it possible to relate with or without contact in a card with the sharing storage and process. Most of progressed smart cards are equipped with a coded expert hard ware that makes it possible to use RSA and DSA Algorithm in the circuit. Today coded smart cards create a pair of key in the circuit in order to prevent more than one copy key. These smart cards are mostly used for digital signature and security identification [6] (see Figure 1).

\subsection{RFID Technology}

RFID system has a kind of usage like magnetic strip installed on credit cards. RFID makes a unique identification for each thing to make it distinguishable from others and the same as information reading possibility on magnetic strip, RFID can be red and in this way it can correct received data (Figure 2).

RFID tag contains the chip part and antenna. Function is like this, at first antenna propagates some radio waves

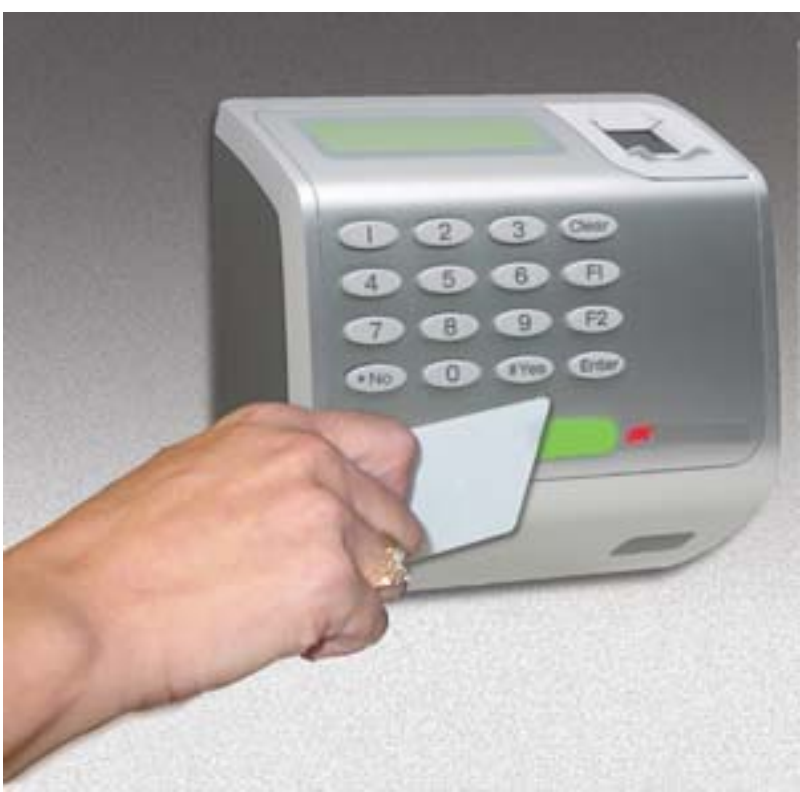

Figure 1. A Sample of smart card.

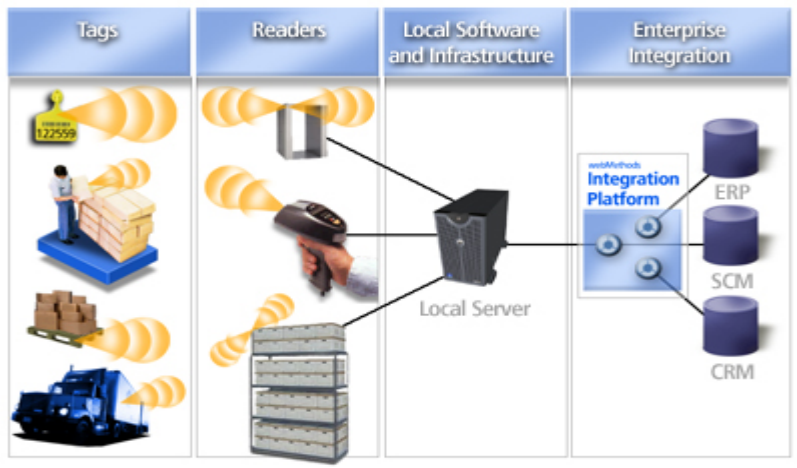

Figure 2. A Sample of RFID technology applications.

in an almost small area. These radio waves which play two roles, are a tool to relate to the RFID tag, and provide needed energy for this communication. When a tag is placed in the produced magnetic field around card reader, stimulator signals of antenna have impact on it and some how they turn the RFID chip on and this chip gives the processed data to the antenna. Data read by the card reader will be sent to the local server and this data will be processed and used in the systems of an organization for different purposes [7].

\subsection{S50 Mifare Cards Specifications}

Used S50 Mifare card are below specification for modeling (Figure 3).

- Data transfer: wireless;

- Function region: more than 100 millimeters;

- Frequency: 56/13 megahertz; 


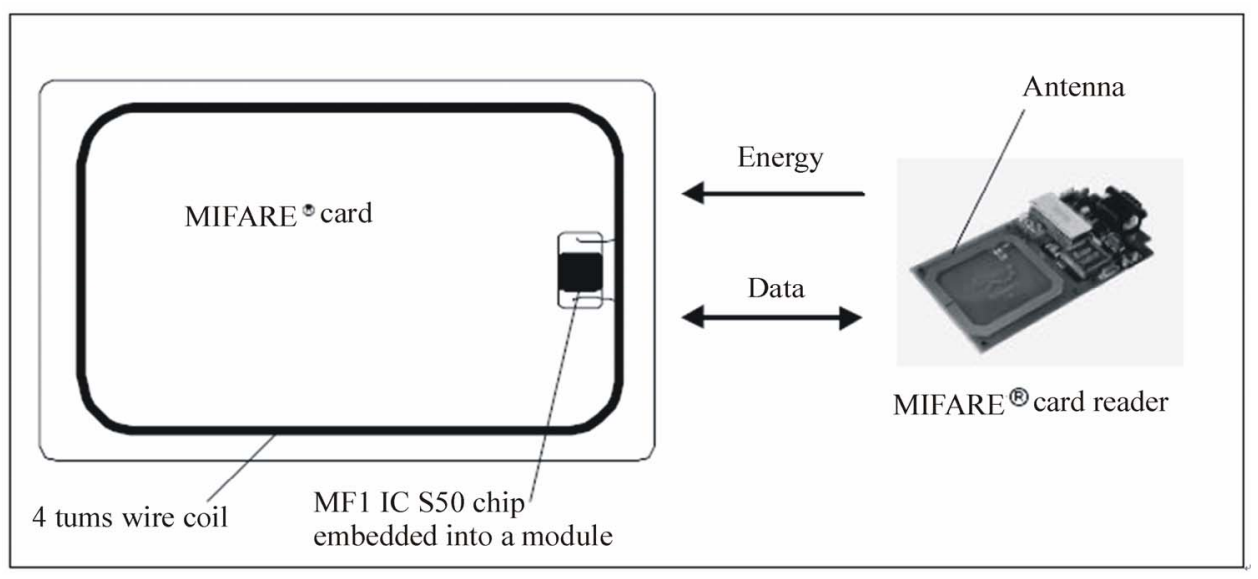

Figure 3. Structure of a sample Mifare card.

- Data transfer rate:106 kilobit per second;

- Has EEPROM with 1 kilo byte capacity;

- Saved data on the card with 10 years expiry;

- Rewrite possibility for 100,000 times;

- 3-steps admission possibility;

- Has two keys to support multi purpose usages;

- Has one serial number $[8,9]$.

\section{Designed System Operation}

The main scenario of the designed system is as below:

The passenger enters the taxi poses his credit card in front of the card reader system available in the taxi ,when credibility of the card is confirmed, card serial number is registered and passenger sits in the taxi and taxi starts moving and by posing the card in front of the system the data is shown. It is necessary to mention that if during driving the possible distance for the passenger, becomes equal to the passed distance from the time passenger got in the taxi, the system alarms "credibility finished". When the passenger gets to the target, again poses his card in front of the system, then passed kilometer is shown and the cost is calculated and deducted from the account. This amount is saved and reported to the central server by the driver, so the driver can receive his money. In this way each driver can send his taxi card reader data to the center at most once a week (sending with card reader itself) and when the center receives the data it sends a response to express reception, and by receiving payments data,center settles equal amount of money to the drivers account (See Figures 4 and 5).

According to the subjects mention till now, it is time

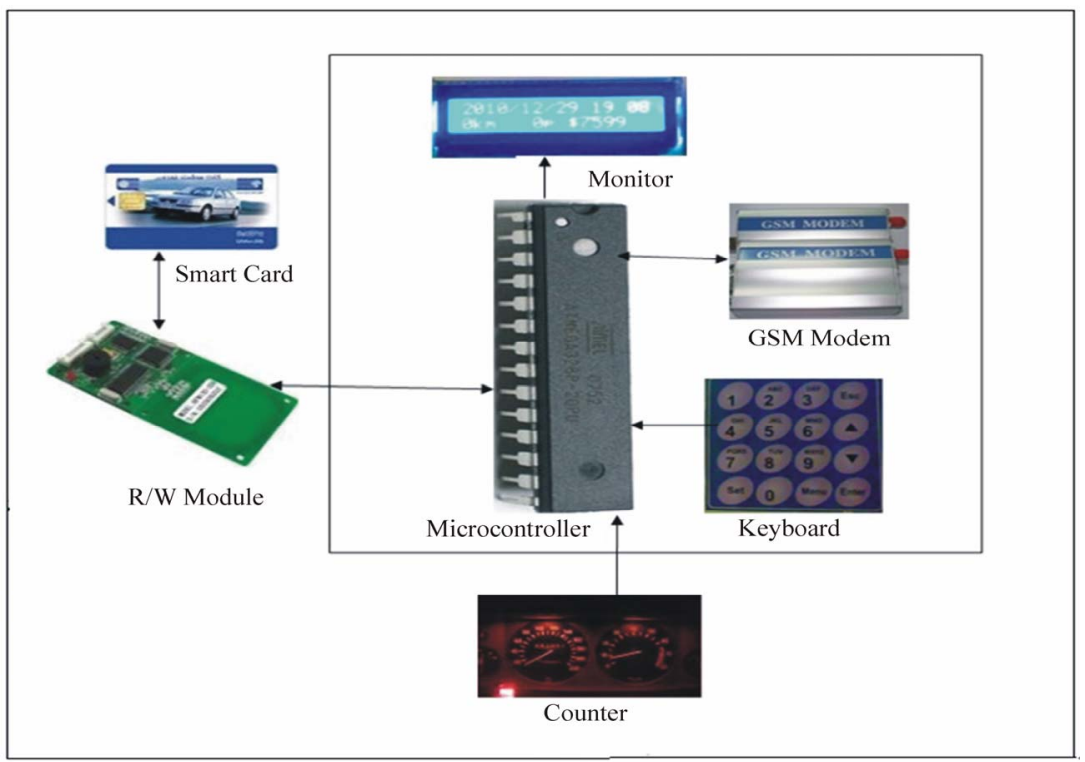

Figure 4. Designed system hardware structure. 


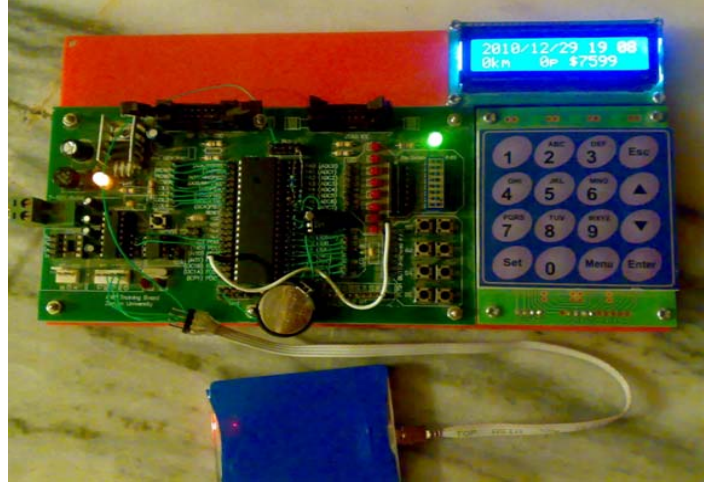

Figure 5. Designed system scheme.

to talk about possibilities of this system:

- To display the money supply and other data for the user;

- To charge the taxi card;

- To count the number of passengers and accept them up to the taxi capacity;

- To calculate the taxi portage by the use of the number of passed kilometers;

- To deduct amount of portage from credibility of the card and store this data in the system for driver to receive his salary later;
- To show error message when the money supply of the card finishes while passenger has not reached the target yet;

- To calculate the cost related to waiting times in the traffic jam;

- To calculate and deduct more portage amount when it is night or early in the morning;

- To display date and time on the main screen;

- Driver can send a message by the use of system and receive his salary electronically (it will be settled to his account).

\section{System Hardware Structure}

The main parts of system hardware structure are: read and write module, connection with center module, keyboard, monitor and a microcontroller (See Figure 6) [10]. When the card is placed in front of read and write module, the connection between these two and through this module data in the card will be transferred to the microcontroller [11]. This data is shown on the monitor screen and is saved in the microcontroller EEROM. Another part of hard ware is for the communication between kilometer counter and microcontroller, so that the number of passed kilometers is sent to micro and according

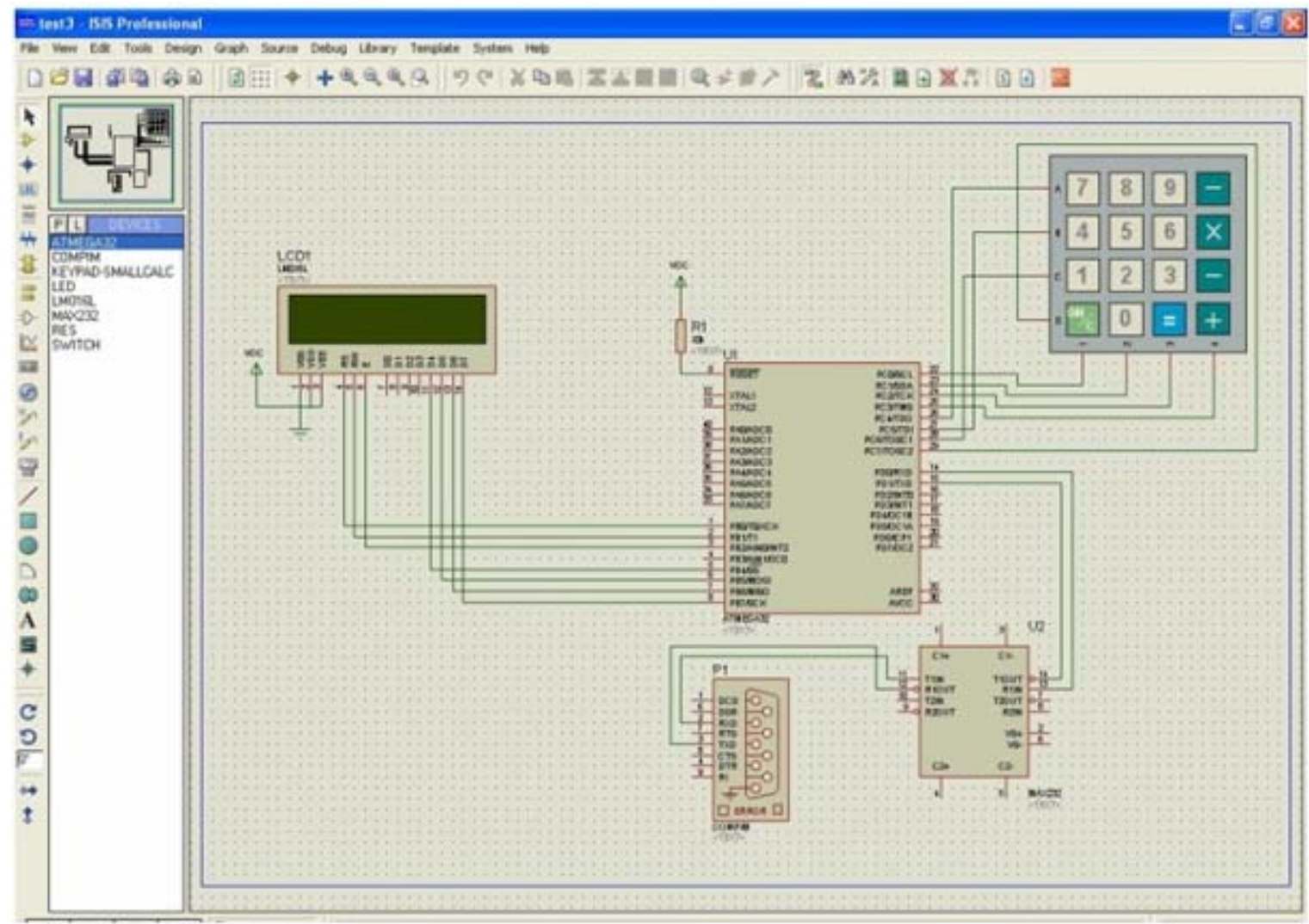

Figure 6. Designed system scheme. 
to the fact that the price of each kilometer is known in the micro, necessary calculations are done and the cost is saved in the EEROM. Next part of hard ware is related to the connection between module with the center and micro, so that the needed data is sent to the main server from the micro memory. It is necessary to mention that all the steps are simulated in the Proteos environment and are programmed in the CodeVision and micro memory is programmed there too.

\section{Conclusions}

This project that took one year to be done led to design a system to pay taxi portage electronically and helped to solve many problems related to portage payment and traffic. In general this system has some prominences such as: not to wasting the time, convenience in use, putting order in the money received from passengers, controlling over the number of passengers considering taxi capacity and creating possibility of bank facilities to drivers.

\section{References}

[1] P. A. Putland and J. Hill, "Electronic Payment Systems," BT Technology Journal, Vol. 15, No. 2, pp. 32-38. doi:10.1023/A:1018676406008

[2] P. A. Putland, C. Ward, A. Jackson and C. Trollope, "Electronic Payment System,” BT Technology Journal, Vol. 17, No. 3, 1999, pp. 67-71. doi:10.1023/A:1009676319984
[3] M. Rader and I. Maghiros, "Electronic Payment System," EPSO Journal, Vol. 15, No. 1, 2001, pp. 1-8.

[4] T. R. Th. and V. Shestalova, "The Price of Cash Revisited," Department of Economics, Tilburg University, Tilburg, March 2001.

[5] B. Mantel, "Why Do Consumers Pay Bills Electronically? An Empirical Analysis,” Federal Reserve Bank of Chicago, Chicago, 2000, pp 30-45.

[6] T. R. Th. and V. Shestalova, "Better Rates Payments" Economic Statistical Journal, Vol. 86, No. 4309, 2001, pp. 411-416.

[7] J. E. Hoag and C. W. Thompson, "Architecting RFID Middleware,” Architecting RFID Middleware, Vol. 10, No. 5, 2006, pp. 88-92.

[8] B. Meng and Q. Xiong, "Research on Electronic Payment Model," Proceeding of the 8th International Conference on, Computer Supported Cooperative Work in Design, Vol. 1, 2004, pp. 597-602.

[9] Z. Y. Lee, H. C. Yu and P. J. Ku, “An Analysis and Comparison of the Different Types of Electronic Payment System," Portland International Conference on Management of Engineering and Technology, Portland, 29 July-2 August 2001, pp. 38-45.

[10] X. Yong and J. D. Liu, "Electronic Payment System Design Based on SET and TTP," 2010 International Conference on E-Business and and E-Government, Guangzhou, 7-9 May 2010, pp. 275-278.

[11] Y. Ling, Y. Xiang and X. Wang, "RSA-Based Secure Electronic Cash Payment System," 2007 IEEE International Conference on Industrial Engineering and Engineering Management, Singapore, 2-4 December 2007, pp. 1898-1902. 